\title{
ROBUST PROCESS-CONTROLLER FOR ND:YAG WELDING
}

\author{
Paper 1806
}

\author{
M.W. de Graaf ${ }^{1}$, J. Olde Benneker ${ }^{2}$, R.G.K.M. Aarts ${ }^{1}$, J. Meijer ${ }^{1}$, J.B. Jonker ${ }^{1}$ \\ ${ }^{1}$ University of Twente - Applied Laser Technology \\ P.O. Box 217 - 7500 AE Enschede - The Netherlands \\ m.w.degraaf@utwente.nl \\ ${ }^{2}$ Netherlands Institute for Metals Research \\ P.O. Box 5008 - 2600 GA Delft - The Netherlands \\ j.oldebenneker@nimr.nl
}

\begin{abstract}
A robust process-controller was developed which maintains a fully penetrated weld. This feedback-controller is robust for various process disturbances that may occur, like variations in sheet thickness, welding velocity, focal position, shielding gas variations, etc. The signals from an optical sensor are used to design a so-called "switching" controller, which enables fully penetrated welding at a minimum amount of laser power. It is shown that the working principle of the controller is applicable to different material types, more specific different steels and stainless steels. Experiments have been carried out to show the ability to cope with varying sheet thickness and welding velocity on FeP04 mild steel. Furthermore a method is described which can be used to easily find a good set of control parameters from a single identification experiment.
\end{abstract}

\section{Introduction}

Full penetration of the weld is an important quality requirement in many laser welding applications. Full penetration can easily be maintained by welding at a relatively low velocity and high laser power. In this case, there is however the risk that the laser power is too high to produce a good quality weld. For economical reasons one would like to use the lowest laser power necessary or highest welding speed close to the boundary where the transition from full penetration to partial penetration occurs. At this boundary there is however always a chance of losing full penetration due to external disturbances like protective window contamination, material batch changes, sheet thickness variation, shielding gas differences, changes in focal position, variations in welding velocity, etc.

Many authors have reported the use of sensors for monitoring the weld pool [1], [2], [3]. Photodiode sensors with different optical filters are used to look at a certain region in the optical spectrum. Often the the plume radiation $(400-800 \mathrm{~nm})$, the thermal radiation (1100-1800nm) and the reflected Nd:YAG radiation $(1064 \mathrm{~nm})$ can be distinguished. A more recent approach to monitoring is the use of CCD or CMOS cameras as they provide a much more detailed image around the keyhole and several interesting features can be extracted directly from the camera image (keyhole area, length of weld pool, width of weld pool, temperature of weld pool etc).

In recent years, process control for laser welding has received a lot of interest as it can cope with varying conditions during laser welding. Many controller designs have been proposed, ranging from standard PID-control to more advanced algorithms. These controllers usually work for one material within a small range of laser power and welding velocity, which makes the applicability in varying industrial conditions difficult. Furthermore the tuning of the control parameters usually is a very timeconsuming job. For control of the welding process often photodiode sensors are used as the bandwidth of the process is rather high and thus a process controller with a fast sampling rate (above $1 \mathrm{Khz}$ ) is needed. Due to the extra processing power that is required for the image feature detection CMOS camera information is rarely used within the control loop at the moment.

Several control approaches can be considered. It has been demonstrated by Postma [4] that the welding depth can be controlled with the use of a standard PI-controller. A second control approach applied by Postma is the use of a "switching" controller [5]. The goal of this controller is to maintain full penetration at a minimum required laser power. The controller maintains the laser power at the transition between partial and full penetration. In terms of robustness to parameter changes it turns out that this controller is not suitable for industrial use. In this paper the "switching" process controller will be extended to make it more robust for deviations that may occur in the process. 


\section{Experimental setup}

The experimental setup is schematically shown in figure 1. A Trumph HL4006D CW Nd:YAG laser is used, which has a maximum power of $4 \mathrm{~kW}$. The laser beam is transmitted through an optical fiber with a diameter of $0.6 \mathrm{~mm}$. A standard Trumph welding head has been used with a $150 \mathrm{~mm}$ focussing lens to obtain a focussed beam diameter of $450 \mu \mathrm{m}$. The focal point has been positioned at the surface of the work piece and is kept constant in all experiments. A lateral shielding gas nozzle is used with Argon as a shielding gas with a flow rate of 15 liter/min.

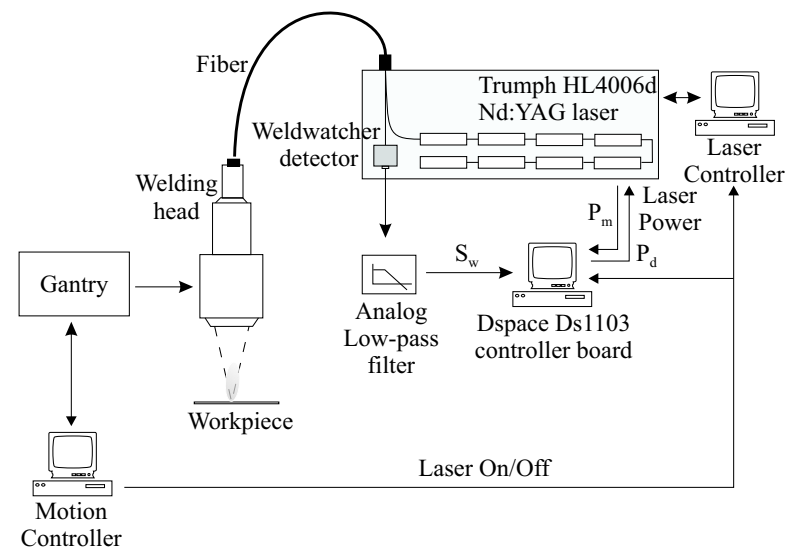

Figure 1: Schematic drawing of the experimental set-up

The process (plume) radiation emitted by the welding process has been detected by a Weldwatcher monitoring system [1]. In this system the process radiation is transmitted through the fiber back to the laser source, where it is measured by a detector. The Weldwatcher system contains an analog low pass filter with a bandwidth of approximately $500 \mathrm{~Hz}$ to filter out high frequency process dynamics.

The welding head is moved with respect to the workpiece using a Gantry system. The laser is switched on and off by the motion controller at the start and end of the motion. A dSpace Ds1103 DSP data acquisition system is used to simultaneously acquire the Weldwatcher signal $S_{w}$ and the measured analog laser power $P_{m}$ at a $2 \mathrm{kHz}$ sampling rate. In all the experiments, the Weldwatcher signal is filtered with a digital 4th order low-pass filter at $100 \mathrm{~Hz}$ at the dSpace system to smoothen the input signal. The process controller implemented in this work is also implemented on the dSpace system. It calculates the desired laser output power $P_{d}$, which is sent to the analog input of the laser. The laser on/off signal from the motion controller is used as a trigger signal for the process controller.

\section{Identification experiment}

To find the relation between the signal level $S_{w}$ of the Weldwatcher system and the laser power, an identification experiment has been carried out. In such an experiment, the laser power is increased from 0 Watt with a certain slope. The results on $0.75 \mathrm{~mm}$ FeP04 mild steel are plotted in figure 2 for speeds of 60,100 and $140 \mathrm{~mm} / \mathrm{s}$. For the speed of $100 \mathrm{~mm} / \mathrm{s}$, several regions have been drawn in this figure. Region I is the conduction welding region, where the signal level increases as a result of an increase in laser power. In region II a keyhole is formed which is still closed at the bottom of the material. When the laser power is further increased a sudden decrease in signal level can be observed, which is the moment at which the keyhole opens at the bottom of the material. In region III a full penetration of the weld is obtained. When the laser power is further increased the weld quality may decrease. Several authors report the sudden drop in signal level [5], [6], [7] when the keyhole comes through. It is expected that a similar signal behavior, with a sudden decrease in signal level at the transition of closed to open keyhole, also occurs for different material types. The sudden drop in signal level only occurs for sufficiently high welding velocities. For lower velocities the signal changes more gradually from increasing slope to decreasing slope at the transition from partial to full penetration.

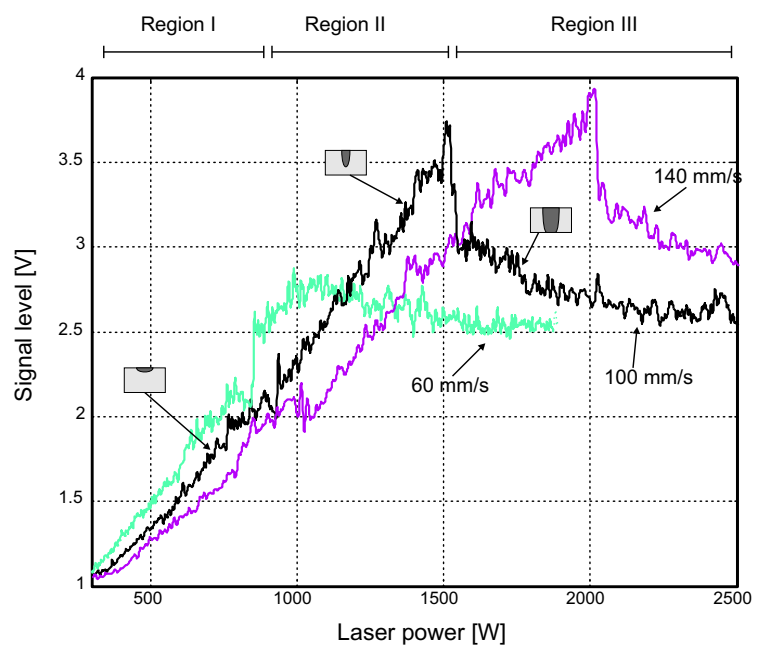

Figure 2: Result of an identification experiment for $\mathrm{FeP04}$

Several control approaches can be considered. It is demonstrated by Postma [4] that the welding depth in region I and II can be controlled with the use of a simple PI-controller. In this region the welding process behaves linearly. For a speed of $100 \mathrm{~mm} / \mathrm{s}$, a signal reference between $1.0 \mathrm{~V}$ and $3.7 \mathrm{~V}$ corresponds to a certain welding depth. A simple control strategy can be used to control 
the welding depth. If the signal level is above the reference value, the laser power should be decreased, if it is below the reference value the laser power should be increased. With this control approach it is very important that the laser power does not enter region III. Or it is likely that the controller will drive the laser power up to its maximum.

\section{Switching control approach}

The second control approach is the use of a "switching" controller [5]. The goal of this controller is to maintain full penetration at a minimum of required laser power. The controller tries to maintain the laser power at the transition between region II and III, where the process exhibits non-linear behavior.

A block diagram of the "switching" controller that maintains full penetration can be found in figure 3 . The sensor signal $S_{w}$ is compared with a threshold value $S_{t r}$. When the on-line signal is higher than the threshold condition, the laser power is increased rapidly to maintain full penetration. When the on-line sensor signal is below the threshold condition the laser power is decreased slowly. The latter keeps the process near the edge of partial penetration. The "switching" controller resembles a PI-controller with the main difference that the proportial gain can be chosen differently, depending on the sign of the difference between $S_{w}$ and $S_{t r}$, i.e. the control gains $K p_{u p}$ and $K p_{\text {down }}$. Furthermore the integral control action uses two different constant rates to increase or decrease the laser power, i.e. the control parameters $K i_{u p}$ and $K i_{\text {down. }}$. This way the controller becomes a non-linear controller, which compensates for the nonlinearities in the transition between partial and full penetration. The controller sets the output power with respect to the nominal laser power $P_{\text {nom }}$.

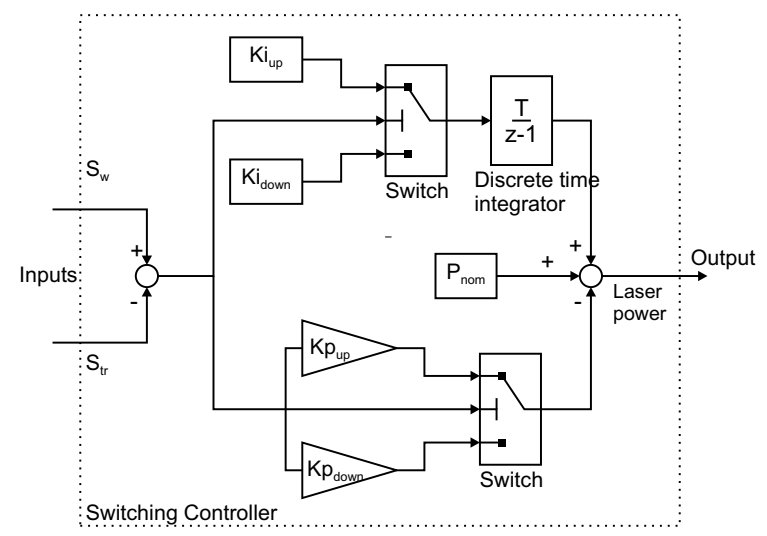

Figure 3: Block diagram of the "switching" controller

Previously a constant threshold value is used, which has some disadvantages with respect to robustness. As can be seen in figure 4 there are two points $A$ and B, where the sensor signal can cross the threshold. B is the correct one at the transition between partial and full penetration. A is at partial penetration at a low laser power. Due to noise on the sensor signal or other disturbances the controller may get in this region. Then the controller will decrease the laser power even more and eventually becomes zero. Under laboratory conditions it is possible to keep the controller in the correct region. In an industrial environment it is doubtful if this can be achieved due to noise and disturbances like material pollution, shielding gas conditions, protective window pollution, etc.

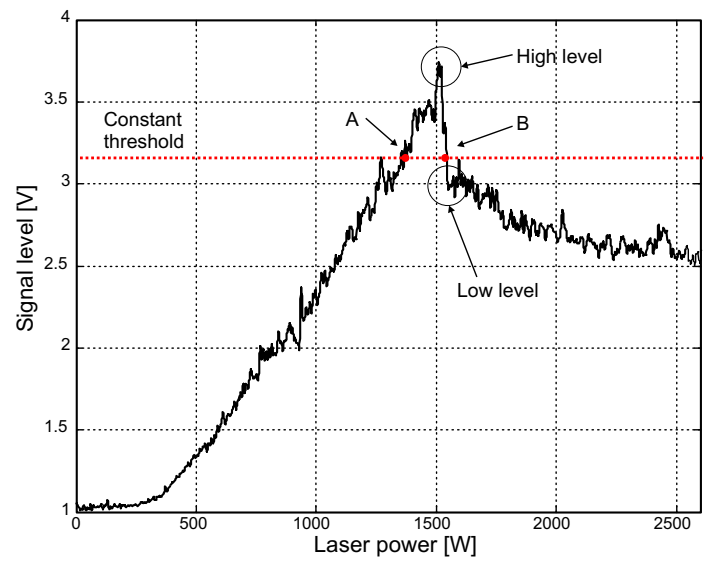

Figure 4: Constant threshold used in Postma's "switching" controller on $0.75 \mathrm{~mm} \mathrm{FeP04}$ at $100 \mathrm{~mm} / \mathrm{s}$

The improvement presented in this work is to apply a linear threshold function instead of a constant value, which dramatically increases the robustness of the "switching" controller. The threshold value $S_{t r}$ will be described as a linear function of the measured laser power $P_{m}$ :

$$
S_{\text {tr }}=\frac{S_{\text {high }}-S_{\text {low }}}{P_{\text {high }}-P_{\text {low }}}\left(P_{m}-P_{\text {low }}\right)+S_{\text {low }},
$$

where $P_{l o w}$ and $P_{\text {high }}$ are two values for laser power and $S_{l o w}$ and $S_{h i g h}$ are two values for the signal level. With well-chosen parameters the sensor signal $S_{w}$ is always above the threshold level $S_{t r}$ at partial penetration and always below $S_{t r}$ at full penetration. There are no regions left where the controller output power drifts to zero or to the maximum output power. In the following section it will be demonstrated how these parameters can be determined from a single identification experiment.

\section{Determining the threshold parameters}

From a number of experiments it was found that the robustness of the controller is mainly determined by the 
threshold function. The parameters of the "switching" controller have an effect on the response of the controller and the ability to reject disturbances, but the stability and robustness are mainly determined by the position of the threshold line, which is determined by just 2 points $\left(P_{\text {low }}, S_{\text {low }}\right)$ and $\left(P_{\text {high }}, S_{\text {high }}\right)$ on the line. In this section it will be shown that they can be determined by carrying out a single identification experiment.

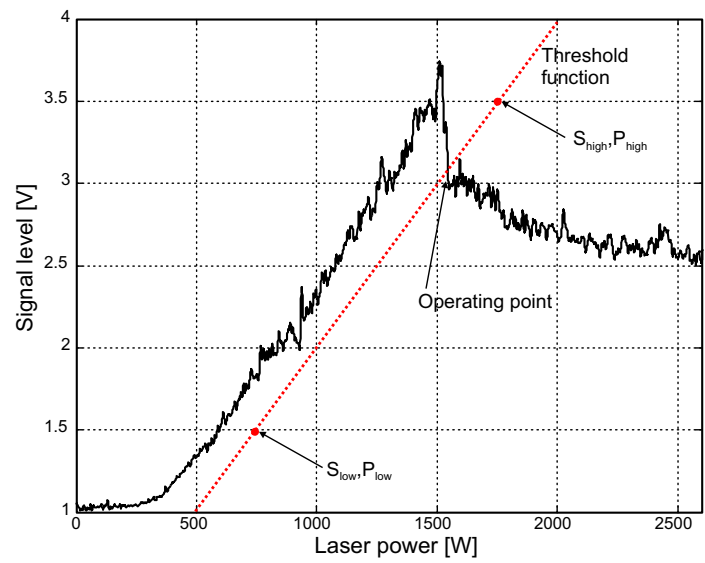

Figure 5: Determining the control parameters from an identification experiment

Such an identification experiment on $0.75 \mathrm{~mm} \mathrm{FeP04}$ at $100 \mathrm{~mm} / \mathrm{s}$ is plotted in figure 5 . The threshold function is drawn as a straight line determined by the threshold parameters. It should be constructed in such a way that:

- The slope of the threshold function is equal or less than the slope of the sensor signal at partial penetration

- The threshold function should cross the signal somewhere between the 'low' signal level and the 'high' level

The operating point of the controller is the crossing of the threshold function with the Weldwatcher signal. The controller always adapts the laser power to reach this operating point. The operating point can be shifted to the right to have some overpower by simply moving the threshold line to the right.

\section{Results}

In this section the results for a number of controlled experiments are presented, which shows the robustness of the controller to various disturbances that may occur in practice. Although the threshold parameters are based on constant process conditions, it is shown that the control approach can cope with many disturbances that may occur.

\begin{tabular}{|l|l|l|l|l|}
\hline Exp. & $K i_{u p}$ & $K i_{\text {down }}$ & $K i_{u p}$ & $K i_{\text {down }}$ \\
\hline A & $0 \mathrm{~W}$ & $100 \mathrm{~W}$ & $10000 \mathrm{~W} / \mathrm{s}$ & $-2000 \mathrm{~W} / \mathrm{s}$ \\
B.1 & $0 \mathrm{~W}$ & $500 \mathrm{~W}$ & $20000 \mathrm{~W} / \mathrm{s}$ & $-4000 \mathrm{~W} / \mathrm{s}$ \\
$\mathrm{B} .2$ & $0 \mathrm{~W}$ & $500 \mathrm{~W}$ & $20000 \mathrm{~W} / \mathrm{s}$ & $-4000 \mathrm{~W} / \mathrm{s}$ \\
$\mathrm{C}$ & $0 \mathrm{~W}$ & $500 \mathrm{~W}$ & $20000 \mathrm{~W} / \mathrm{s}$ & $4000 \mathrm{~W} / \mathrm{s}$ \\
\hline
\end{tabular}

Table 1: Control parameters

\begin{tabular}{|l|l|l|l|l|}
\hline Exp. & $P_{\text {low }}$ & $S_{\text {low }}$ & $P_{\text {high }}$ & $S_{\text {high }}$ \\
\hline $\mathrm{A}$ & $500 \mathrm{~W}$ & $1 \mathrm{~V}$ & $1500 \mathrm{~W}$ & $3 \mathrm{~V}$ \\
B.1 & $500 \mathrm{~W}$ & $1 \mathrm{~V}$ & $1500 \mathrm{~W}$ & $3 \mathrm{~V}$ \\
B.2 & $700 \mathrm{~W}$ & $1 \mathrm{~V}$ & $1800 \mathrm{~W}$ & $3 \mathrm{~V}$ \\
B.3 & $700 \mathrm{~W}$ & $1 \mathrm{~V}$ & $1800 \mathrm{~W}$ & $3 \mathrm{~V}$ \\
\hline
\end{tabular}

Table 2: Threshold parameters

The control parameters and the threshold parameters used in each experiment are given in tables 1 and 2 .

\section{A. Minimum required laser power}

First an experiment has been carried out to show the ability of the controller to find the minimum of required laser power without disturbances. Therefore a bead-on-plate weld at $100 \mathrm{~mm} / \mathrm{s}$ has been carried out on a sheet of 0.75 $\mathrm{mm}$ FeP04. The starting power $P_{\text {nom }}$ is 1800 Watt and control starts after $0.3 \mathrm{~s}$. The measured laser power is plotted in figure 6 .

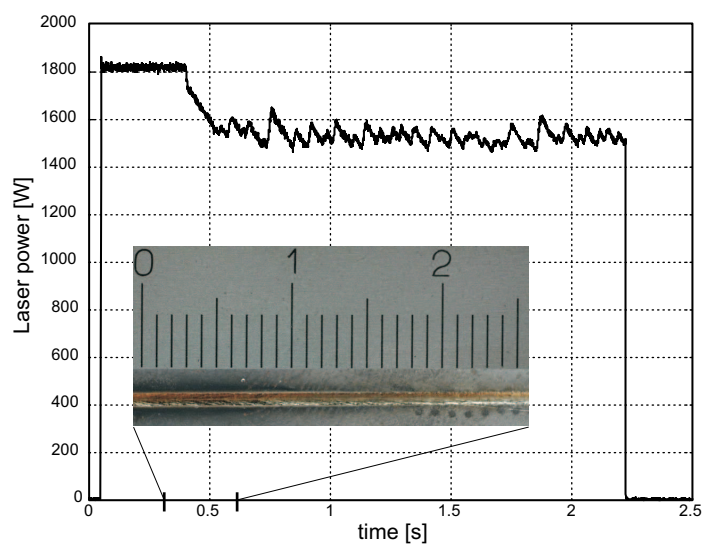

Figure 6: Controlled experiment (A) to find the minimum required laser power

As expected the laser power decreases from $1800 \mathrm{~W}$ to a stationary value of somewhat less than $1600 \mathrm{~W}$. Furthermore visual observation showed that a very smooth weld was obtained, the fluctuations in the output of the controller could not be seen in the weld. 


\section{B. Sheet thickness variation}

As a first disturbance for the controller a sheet of 0.75 $\mathrm{mm}$ FeP04 was locally decreased to a thickness of 0.5 $\mathrm{mm}$ (figure 7). The disturbance was at the bottom side of the sheet to obtain the focal position on the surface. A velocity of $100 \mathrm{~mm} / \mathrm{s}$ was used. The measured laser power is plotted in figure 8 .

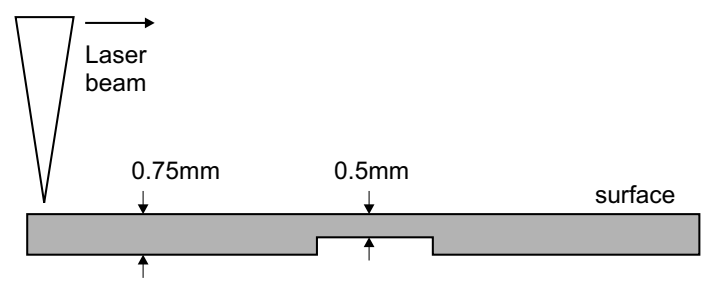

Figure 7: $0.75 \mathrm{~mm}$ sheet with local decrease to $0.5 \mathrm{~mm}$

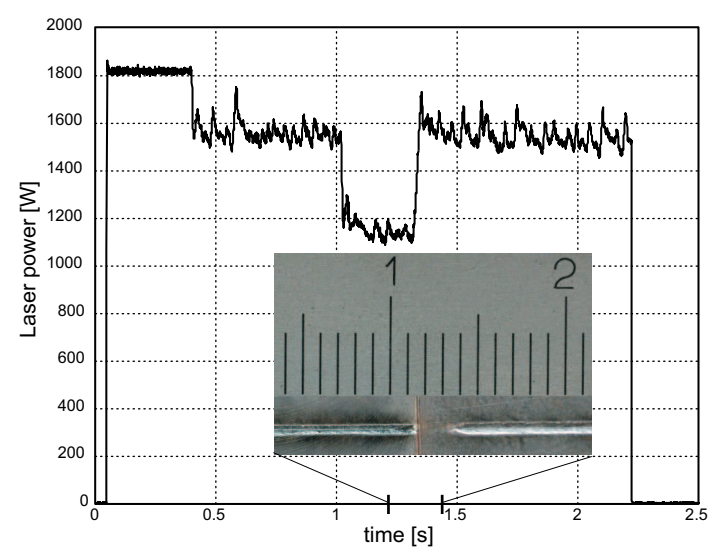

Figure 8: Controlled experiment (B.1) with local decrease in sheet thickness

Control starts again at $0.3 \mathrm{~s}$ and the laser power goes again to a stationary value around $1550 \mathrm{~W}$. At the local decrease in sheet thickness the laser power quickly decreases to $1150 \mathrm{~W}$. After the disturbance has passed the laser power returns to its stationary value of $1550 \mathrm{~W}$. Because of the sudden decrease in sheet thickness the controller needs some time to react to the new situation. It takes about $2 \mathrm{~mm}$ before the controller output is adapted to the lower power. At $100 \mathrm{~mm} / \mathrm{s}$ this corresponds to a settling time of $20 \mathrm{~ms}$.

The controller can cope with an increase in sheet thickness. To demonstrate this a bead-on-plate weld has been carried out on a sheet with a local increase in sheet thickness from $0.75 \mathrm{~mm}$ to $1.5 \mathrm{~mm}$ (figure 9).

A velocity of $100 \mathrm{~mm} / \mathrm{s}$ was used again. The measured laser power is plotted in figure 10 . The threshold values were adapted in such a way that full penetration was maintaned for both the $0.75 \mathrm{~mm}$ and $1.5 \mathrm{~mm}$ sheet thickness.

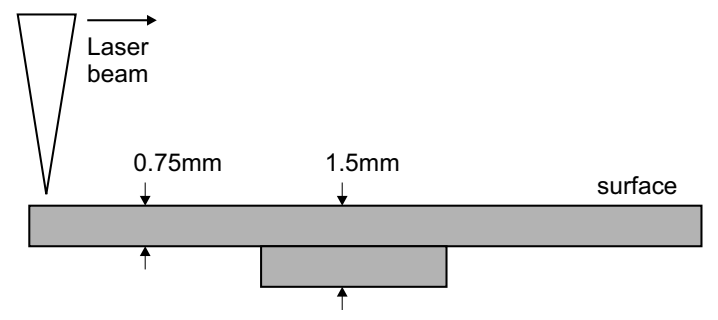

Figure 9: $0.75 \mathrm{~mm}$ sheet with local increase to $1.5 \mathrm{~mm}$

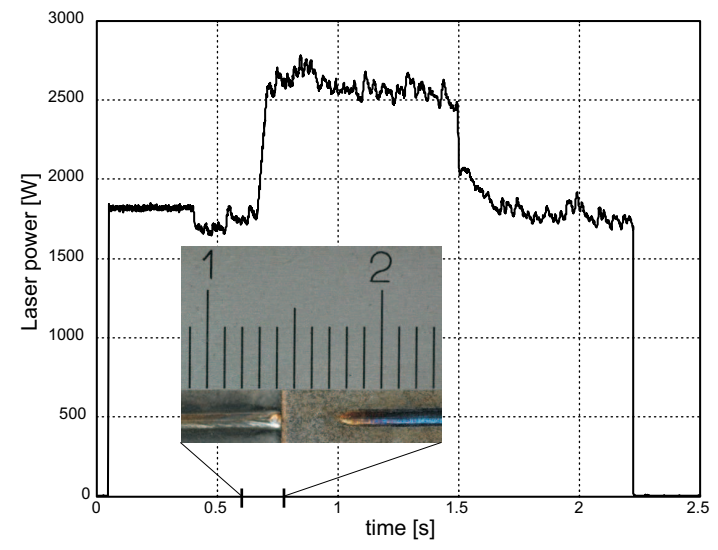

Figure 10: Controlled experiment (B.2) with local increase in sheet thickness

Control starts at $0.3 \mathrm{~s}$ and the laser power goes to a stationary value around $1700 \mathrm{~W}$. At the local increase in sheet thickness the laser power quickly increases to maintain full penetration. After the disturbance has passed the laser power returns to its stationary value. This experiment shows that the controller needs about $30 \mathrm{~ms}$ to react to the sudden disturbances in sheet thickness.

\section{Welding velocity}

The proposed controller is also able to cope with variations in welding speed. To demonstrate this behavior a bead-on-plate weld has been carried out where the velocity increases from $100 \mathrm{~mm} / \mathrm{s}$ to $120 \mathrm{~mm} / \mathrm{s}$ half way the weld. The measured laser power is plotted in figure 11 .

At the increase in velocity, the laser power increases as well to maintain full penetration. Visual inspection of the weld shows that full penetration was indeed maintained.

\section{Robustness to material choice}

The possibilities on using this control approach for maintaining full penetration on different material types than FeP04 depend very much on the presence of the sudden drop in signal level that was observed from an identification experiment. Therefore in this section identification 


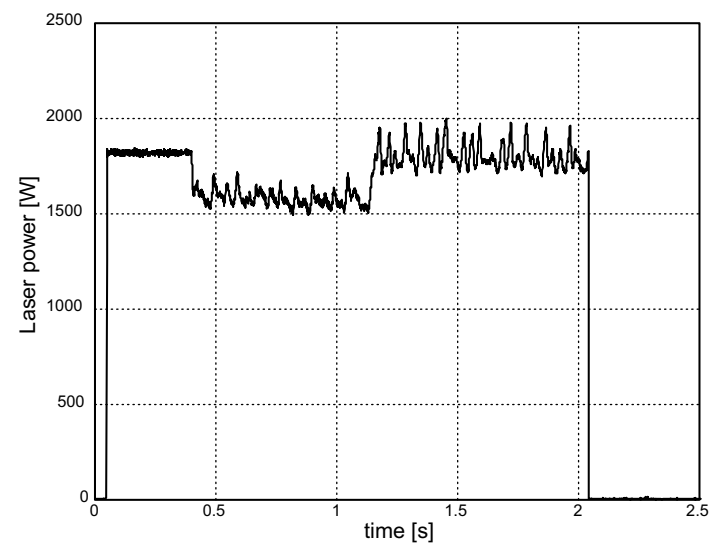

Figure 11: Controlled experiment (C) with changing welding velocity (100 to $120 \mathrm{~mm} / \mathrm{s}$ )

experiments have been carried out for different materials to see if a similar behavior can be observed. An identification experiment has been carried out on $1 \mathrm{~mm}$ AISI304 stainless steel at a speed of $100 \mathrm{~mm} / \mathrm{s}$. The results of this experiment can be found in figure 12 . Very similar results with the identification on FeP04 can be seen. Especially the transition between partial and full penetration results in a clear sudden decrease in signal level. Therefore it is expected that the proposed process controller is also useful for this material.

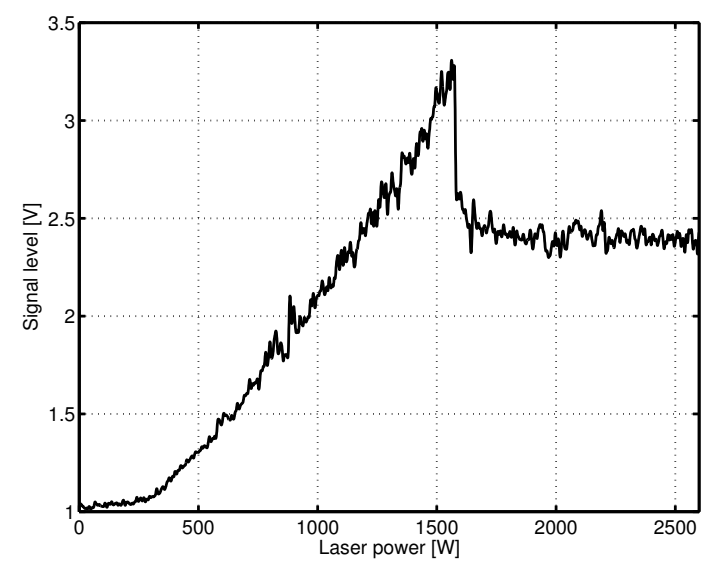

Figure 12: Identification experiment for $1 \mathrm{~mm}$ AISI304 Stainless Steel at $100 \mathrm{~mm} / \mathrm{s}$

An identification experiment has also been carried out on $1 \mathrm{~mm}$ AA5182 Aluminium, also at a speed of 100 $\mathrm{mm} / \mathrm{s}$. The results for this experiment are found in figure 13. Compared to FeP04 and AISI304 a different behavior can be noticed. Although full penetration is achieved at around $1500 \mathrm{~W}$, the sudden decrease in signal level can't be observed at all. The signal keeps on increasing if the laser power is increased in the full penetration regime. Furthermore it can be noticed that the sig- nal level is much lower for this material. Therefore the proposed controller can't be used for this material at the moment. Process control for this material is a topic for further research.

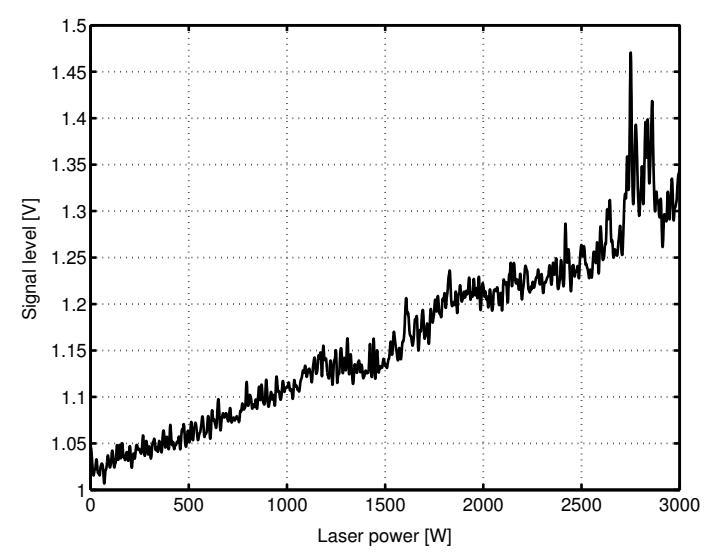

Figure 13: Identification experiment for $1 \mathrm{~mm}$ AA5182 Aluminium at $100 \mathrm{~mm} / \mathrm{s}$

\section{Conclusions and discussion}

In this paper a process controller is presented which controls the laser power. It maintains full penetration at a minimum amount of laser power. The main advantage of this controller compared to previous process controllers in literature is its robustness. It was shown for FeP04 that the controller can handle changes in sheet thickness and welding velocity and adapts the laser power accordingly. The controller proved to be robust for both a decrease and an increase in sheet thickness. A change in welding velocity proved to be no problem as well.

Furthermore identification experiments have been carried out on AA5182 and AISI304, which shows that the characteristic drop in the measured sensor signal at the transition between partial and full penetration also occurs for stainless steel. It is expected that the controller can be applied to a large series of steels and stainless steels. An identification experiment on aluminum showed that this material behaves quite differently. Possibly different optical filters may help to increase the applicability of the controller on aluminium. Further investigations need to be carried out on aluminium and its alloys.

The controller settling time proved to be too low for applications where very sudden disturbances occur. It is expected that a faster response to disturbances can still be obtained by improving the control parameters and by using different filtering methods. This will be part of further work. 


\section{Acknowledgements}

Part of this research was carried out under the project number MC8.00073 in the framework of the Strategic Research Program of the Netherlands Institute for Metals Research in the Netherlands (www.nimr.nl).

\section{References}

[1] R. Güttler. Sensor detects faults in the keyhole. Opto \& Laser Europe, October 1998.

[2] J. Beersiek, P. Poprawe, W. Schulz, H. Gu, R.E. Mueller, and W.W. Duley. On-line monitoring of penetration depth in laser beam welding. In Proc. of ICALEO '97, 1997.

[3] S. Postma, R.G.K.M. Aarts, and J. Meijer. Penetration detection in nd:yag laser welding using optical sensors. In Proceedings of the 9th International Conference on Sheet Metal, Leuven, Belgium, 2001.

[4] S. Postma. Weld pool control in Nd:YAG Laser welding. $\mathrm{PhD}$ thesis, University of Twente, 2003.

[5] S. Postma, R.G.K.M. Aarts, J. Meijer, and J.B. Jonker. Feedback control for optimal production speed in laser beam welding of mild steel. In Proc. of ICALEO '02, 2002.

[6] P.G. Sanders, J.S. Keske, G. Kornecki, and K.H. Leong. Capabilities of infrared weld monitor. In Proc. of ICALEO '97, 1997.

[7] B.N. Bad'Yanov and A.V. Polyakov. Control of laser welding in real time regime. Welding International, 46:814-817, 1999.

\section{Meet the authors}

Menno de Graaf studied Mechanical Engineering at the University of Twente and received his M.Sc degree in 2002. He is currently working as a $\mathrm{PhD}$ researcher at the Chair of Applied Laser Technology of the University of Twente on the topic of integration of sensors in a robotic laser welding unit.

Jeroen Olde Benneker studied Mechanical Engineering at the University of Twente and received his M.Sc degree in 1996. He is currently working as a consultant knowledge transfer at the Netherlands Institute for Metals Research.

Johan Meijer is professor in the field of Applied Laser Technology at the University of Twente. Ronald Aarts is a senior researcher at the University of Twente and Ben
Jonker is professor and chairman of the Mechanical Automation group at the faculty of Engineering Technology at the University of Twente. 